\title{
A novel chemical-combination screen in zebrafish identifies epigenetic small molecule candidates for the treatment of Duchenne muscular dystrophy
}

Gist H. Farr III ${ }^{1}$, Melanie Morris ${ }^{1,2}$, Arianna Gomez ${ }^{1,3,4}$, Thao Pham ${ }^{1,3}$, Elisabeth Kilroy ${ }^{5}$, Elizabeth U. Parker ${ }^{1,2}$, Shery Said ${ }^{1}$, Clarissa Henry ${ }^{6}$ and Lisa Maves ${ }^{1,4^{*}}$ (i)

\begin{abstract}
Background: Duchenne muscular dystrophy (DMD) is a severe neuromuscular disorder and is one of the most common muscular dystrophies. There are currently few effective therapies to treat the disease, although many small-molecule approaches are being pursued. Certain histone deacetylase inhibitors (HDACi) have been shown to ameliorate DMD phenotypes in mouse and zebrafish animal models. The HDACi givinostat has shown promise for DMD in clinical trials. However, beyond a small group of HDACi, other classes of epigenetic small molecules have not been broadly and systematically studied for their benefits for DMD.

Methods: We used an established animal model for DMD, the zebrafish dmd mutant strain sapje. A commercially available library of epigenetic small molecules was used to treat embryonic-larval stages of dmd mutant zebrafish. We used a quantitative muscle birefringence assay in order to assess and compare the effects of small-molecule treatments on dmd mutant zebrafish skeletal muscle structure.

Results: We performed a novel chemical-combination screen of a library of epigenetic compounds using the zebrafish $d m d$ model. We identified candidate pools of epigenetic compounds that improve skeletal muscle structure in dmd mutant zebrafish. We then identified a specific combination of two HDACi compounds, oxamflatin and salermide, that ameliorated $d m d$ mutant zebrafish skeletal muscle degeneration. We validated the effects of oxamflatin and salermide on $d m d$ mutant zebrafish in an independent laboratory. Furthermore, we showed that the combination of oxamflatin and salermide caused increased levels of histone $\mathrm{H} 4$ acetylation in zebrafish larvae.

Conclusions: Our results provide novel, effective methods for performing a combination of small-molecule screen in zebrafish. Our results also add to the growing evidence that epigenetic small molecules may be promising candidates for treating DMD.
\end{abstract}

Keywords: Chemical screen, Duchenne muscular dystrophy, Epigenetic small molecules, HDAC inhibitors, Zebrafish

\footnotetext{
*Correspondence: Imaves@u.washington.edu

${ }^{1}$ Center for Developmental Biology and Regenerative Medicine, Seattle

Children's Research Institute, Seattle, WA, USA

${ }^{4}$ Department of Pediatrics, University of Washington, Seattle, WA, USA

Full list of author information is available at the end of the article
}

C C The Author(s). 2020 Open Access This article is licensed under a Creative Commons Attribution 4.0 International License, which permits use, sharing, adaptation, distribution and reproduction in any medium or format, as long as you give appropriate credit to the original author(s) and the source, provide a link to the Creative Commons licence, and indicate if changes were made. The images or other third party material in this article are included in the article's Creative Commons licence, unless indicated otherwise in a credit line to the material. If material is not included in the article's Creative Commons licence and your intended use is not permitted by statutory regulation or exceeds the permitted use, you will need to obtain permission directly from the copyright holder. To view a copy of this licence, visit http://creativecommons.org/licenses/by/4.0/ The Creative Commons Public Domain Dedication waiver (http://creativecommons.org/publicdomain/zero/1.0/) applies to the data made available in this article, unless otherwise stated in a credit line to the data. 


\section{Background}

Duchenne muscular dystrophy (DMD) is a severe neuromuscular disorder caused by mutations in the X-linked $D M D$ gene, which encodes the protein dystrophin $[1,2]$. Dystrophin is a key component of the dystrophinassociated protein complex (DAPC), a multi-protein assembly localized at the sarcolemma of muscle cells $[3,4]$. Dystrophin and the DAPC serve a structural role in muscle cells, functioning as a stabilizing link between the cytoskeleton and extracellular matrix during muscle fiber contraction [5-7]. Dystrophin and the DAPC also regulate signaling pathways such as nitric oxide production and $\mathrm{Ca}^{2+}$ entry [6-9]. Loss of dystrophin and DAPC function makes muscle cell membranes susceptible to contractioninduced damage and leads to progressive calcium dysregulation, satellite cell dysfunction, inflammation, fibrosis, and necrosis [7, 10-13].

DMD is the most common type of muscular dystrophy, affecting approximately 1 in 3500-5000 male births $[14,15]$. It first presents as motor difficulties in early childhood and progresses rapidly, leaving most affected boys in need of a wheelchair in their early teens and in need of respiratory aid in their 20s [16]. The disease is usually fatal in the third or fourth decade due to respiratory or cardiovascular failure.

Treatment options for DMD are still quite limited. The current standard of care is corticosteroid treatment, which delays the progression of muscle dysfunction but has serious side effects [16-18]. Besides corticosteroids, two other drugs are approved for a subset of DMD patients: eteplirsen, an exon-skipping oligonucleotide drug for patients with $D M D$ exon 51 mutations, has received accelerated FDA approval in the USA, and ataluren, a stop codon read-through drug, has conditional approval in Europe for patients with $D M D$ nonsense mutations $[16,19]$. DMD gene therapy and gene editing approaches are very promising but face many challenges [20-23]. Many small-molecule approaches are being identified that could benefit DMD by modulating different pathological mechanisms downstream of the dystrophin mutation $[7,16,19,24]$. A current view in the DMD field is that a combination of therapies targeting different pathological mechanisms may ultimately be most beneficial for patients $[19,24]$.

Histone deacetylase inhibitors (HDACi) are one class of small molecules that have shown promise in mouse and zebrafish DMD animal models and in DMD clinical trials (ClinicalTrials.gov NCT02851797, NCT03373968) [25-28]. HDACi are examples of "epigenetic small molecules," compounds that target chromatin modifications and transcriptional regulators. Histone acetylation, which is often linked with open chromatin and active transcription, is one example of an epigenetic modification and is the target of many HDACi [29]. There are four different classes of histone deacetylase (HDAC) proteins, with different functions and tissue expression patterns, and different HDACi often selectively inhibit specific HDACs [30]. Studies in the $m d x$ mouse model of DMD have identified epigenetic mechanisms involved in the pathogenesis of DMD, including constitutive activation of HDAC2 and dysregulation of certain histone modifications, leading to downstream transcriptional perturbations [31-34]. Dystrophin and the DAPC can regulate chromatin signaling through nitric oxide inhibition of HDAC2 $[25,32]$. HDACi that have been shown to ameliorate pathology in the $m d x$ mouse are the panHDACi Trichostatin A (TSA), valproic acid, phenylbutyrate, SAHA, and givinostat (ITF 2357), and the class I HDACi MS-275 [25, 28, 35-37]. TSA also ameliorates the zebrafish $d m d$ mutant $[27,38]$, and givinostat has shown promise in DMD clinical trials [26]. However, beyond these HDACi, other classes of epigenetic small molecules have not been broadly and systematically tested in DMD models for their potential benefits for DMD.

Zebrafish are an outstanding animal model for DMD [39-43]. A zebrafish $d m d$ mutant strain, $d m d^{t a 222 a}$, also known as sapje, has a nonsense mutation in exon 4 and is a dystrophin-deficient model of DMD [39, 41, 44]. The zebrafish $d m d^{\text {ta222a }}$ mutation is autosomal recessive, and, following a Mendelian ratio, approximately 25\% of the offspring from a heterozygous $d m d+/-$ cross exhibit degenerative muscle lesions by about 3 days postfertilization. dmd mutant zebrafish exhibit many aspects of human DMD pathology; in particular, skeletal muscle fibrosis and inflammation, including infiltration of mononuclear cells [40, 41].

dmd mutant zebrafish offer several advantages for screening and evaluating small molecule therapies [42, 43]. Zebrafish eggs can be rapidly produced in large numbers, and the resulting embryos readily absorb small molecules. Because skeletal muscle lesions can be observed within 3-4 days of development, $d m d$ mutant zebrafish are amenable to rapid and high-throughput screening. An exceptional range of approaches are available for assessing treatment outcomes in $d m d$ mutant zebrafish, including assays of skeletal muscle structure and function as well as survival $[42,43,45,46]$. In particular, muscle structure can easily be observed in zebrafish larvae using polarized light birefringence techniques [44, 45, 47]. Large-scale chemical screens have highlighted the potential of $d m d$ mutant zebrafish for identifying new therapeutic compounds and targets as well as for understanding the molecular mechanisms behind DMD [45, 48, 49]. These large-scale screens, which applied either pools of eight chemicals or individual chemicals to $d$ md mutant zebrafish, have tested over 4000 compounds and identified 25 positive hits [43]. In addition, some small molecules, such as TSA, have been 
shown to ameliorate both $d m d$ mutant zebrafish and $m d x$ mice [27, 45, 50-52]. Because of this strong conservation, insight from zebrafish can inform our understanding of human DMD disease, while also taking advantage of the utility of high-throughput analysis.

Here, we performed a pilot screen of the commercially available Cayman Chemical Epigenetics Screening Library to identify additional epigenetic small molecules that could improve the $d m d$ mutant zebrafish muscle phenotype. We identified candidate pools of epigenetic compounds that improve skeletal muscle structure in $d m d$ mutant zebrafish. We then identified a specific combination of two HDACi compounds, oxamflatin and salermide, that ameliorated dmd mutant zebrafish skeletal muscle degeneration. We validated the effects of oxamflatin and salermide on $d m d$ mutant zebrafish in an independent laboratory. Furthermore, we showed that the combination of oxamflatin and salermide caused increased levels of histone $\mathrm{H} 4$ acetylation in zebrafish larvae. Overall, our study provides novel and effective methods for performing a pooled chemical-combination screen and gives further evidence that epigenetic small molecules may be good candidates for successfully treating DMD.

\section{Methods}

\section{Zebrafish husbandry}

All experiments involving live zebrafish (Danio rerio) were carried out in compliance with Seattle Children's Research Institute's and the University of Maine's Institutional Animal Care and Use Committee guidelines. Zebrafish were raised and staged as previously described [53]. Time (hpf or dpf) refers to hours or days post-fertilization at $28.5^{\circ} \mathrm{C}$. Eggs were collected from 20-30-min spawning periods and raised in Petri dishes in ICS water $(300 \mathrm{mg}$ Instant Ocean/L, $0.56 \mathrm{mM} \mathrm{CaCl} 2,1.2 \mathrm{mM} \mathrm{NaHCO} 3)$, in a dark $28.5^{\circ} \mathrm{C}$ incubator, up to $5 \mathrm{dpf}$. After $5 \mathrm{dpf}$, fish were maintained on a recirculating water system (Aquaneering) under a 14-h on, 10-h off light cycle. From 6-30 dpf, fish were raised in $2.8 \mathrm{~L}$ tanks with a density of no more than 50 fish per tank and fed a standard diet of paramecia (Carolina) one time per day and Zeigler AP100 dry larval diet two times per day. From $30 \mathrm{dpf}$ on, fish were raised in $6 \mathrm{~L}$ tanks with a density of no more than 50 fish per tank and fed a standard diet of Artemia nauplii (Brine Shrimp Direct) and Zeigler adult zebrafish feed, each two times per day. The wild-type stock and genetic background used was AB. The zebrafish $d m d^{t a 222 a} \mathrm{mu}-$ tant line (also known as sapje; hereafter, referred to as $d m d$ ) has been described and is a null allele [39, 44]. $d m d^{t a 222 a}$ genotyping was performed as previously described [54].

\section{Small molecules}

Epigenetic small molecule library screening was performed using the Cayman Chemical Epigenetics Screening Library (Item No. 11076, Batch No. 0455098). The library was received as $10 \mathrm{mM}$ stocks of each chemical dissolved in dimethyl sulfoxide (DMSO). The composition of the Cayman Chemical Epigenetics Screening Library can vary. The version we obtained contained 94 chemicals distributed over two 96-well plates. The identities of the chemicals and plate layouts are shown in Table 1 and Fig. 1a. Additional chemicals were purchased individually from Cayman Chemical and were dissolved in DMSO (Sigma).

\section{Small molecule treatments}

To test toxicity and assess working doses of the Epigenetics Screening Library chemicals, wild-type AB embryos were treated with individual chemicals beginning at 4 hpf. Embryos were not dechorionated prior to these dose tests. Three concentrations of each chemical were tested: $10 \mu \mathrm{M}, 1 \mu \mathrm{M}$, and $100 \mathrm{nM}$. Embryos were treated with small molecules with $1 \%$ DMSO in embryo medium (EM; $\quad 14.97 \mathrm{mM} \quad \mathrm{NaCl}, \quad 0.50 \mathrm{mM} \quad \mathrm{KCl}, \quad 0.98 \mathrm{mM}$ $\mathrm{CaCl}_{2} .2 \mathrm{H} 2 \mathrm{O}, 0.15 \mathrm{mM} \mathrm{KH}_{2} \mathrm{PO}_{4}, 0.99 \mathrm{mM} \mathrm{MgSO}_{4} .7 \mathrm{H} 2 \mathrm{O}$, $\left.0.05 \mathrm{mM} \mathrm{Na}_{2} \mathrm{HPO}_{4}, 0.83 \mathrm{mM} \mathrm{NaHCO} 3\right)$, or with $1 \%$ DMSO in EM as a vehicle control. One chemical from the library, JGB1741, exhibited precipitation from EM at $10 \mu \mathrm{M}$ but not at lower concentrations; all other chemicals appeared soluble with DMSO in EM. Twelve-well plates were used for treatments; each well contained 25 embryos and $3 \mathrm{~mL}$ of chemical treatment or vehicle control. The treatment media was changed every $24 \mathrm{~h}$ until $4 \mathrm{dpf}$. Over the 4-day treatments, developmental abnormalities and survival were noted. Most chemical treatments showed little or no effect on embryo health and survival at $1 \mu \mathrm{M}$ doses, so we determined $1 \mu \mathrm{M}$ to be the working dose for pooled screening for most of the library chemicals. C646 and apicidin were toxic and caused embryo lethality at $1 \mu \mathrm{M}$ but not at $100 \mathrm{nM}$, so these compounds were used at $100 \mathrm{nM}$. CAY10669 exhibited strong toxicity and GSK-J1 is reported to be non-cell permeable, so these chemicals were removed from chemical pool screening. (-)-JQ1, MI-nc, GSK-J2, and GSK-J5 are designated as negative control compounds in the library and thus were also removed from chemical pool screening.

For chemical screening and $d m d$ rescue tests, embryos from $d m d+/-$ crosses were used. At about $24 \mathrm{hpf}$, the chorions were manually removed from the embryos, and embryos were sorted into wells of 12-well plates for treatments. Each well contained 25 embryos and $3 \mathrm{~mL}$ of chemical treatment or vehicle control. 1000X stocks of chemicals were made in DMSO just prior to each treatment experiment. Embryos were treated with chemicals 
Table 1 Cayman chemical epigenetics screening library

\begin{tabular}{|c|c|c|c|}
\hline \multicolumn{2}{|c|}{ Plate 1} & \multicolumn{2}{|c|}{ Plate 2} \\
\hline Well & Small molecule & Well & Small molecule \\
\hline A2 & 3-amino benzamide & $A 2$ & AGK2 \\
\hline A3 & SB 939 & A3 & CAY10603 \\
\hline A4 & PCl 34051 & A4 & Chaetocin \\
\hline A5 & 4-iodo-SAHA & A5 & Splitomicin \\
\hline A6 & Sirtinol & A6 & $\mathrm{CBHA}$ \\
\hline A7 & C646 & A7 & M 344 \\
\hline A8 & Ellagic acid & A8 & Oxamflatin \\
\hline A9 & Scriptaid & A9 & Salermide \\
\hline $\mathrm{A} 10$ & Suberohydroxamic acid & $\mathrm{A} 10$ & Mirin \\
\hline A11 & Apicidin & A11 & Pimelic diphenylamide 106 \\
\hline B2 & UNC0321 (trifluoroacetate salt) & B2 & (S)-HDAC-42 \\
\hline B3 & (-)-Neplanocin A & B3 & MS-275 \\
\hline B4 & Cl-Amidine & B4 & HNHA \\
\hline B5 & F-Amidine (trifluoroacetate salt) & B5 & RG-108 \\
\hline B6 & JGB1741 & B6 & 2',3',5'-triacetyl-5-azacytidine \\
\hline B7 & UNC0638 & B7 & S-Adenosylhomocysteine \\
\hline B8 & Phthalazinone pyrazole & B8 & UNC0224 \\
\hline B9 & Isoliquiritigenin & B9 & Chidamide \\
\hline B10 & CCG-100602 & B10 & 3-Deazaneplanocin A \\
\hline B11 & CAY10669 & B11 & Sinefungin \\
\hline C2 & Zebularine & C2 & Pyroxamide \\
\hline C3 & Delphinidin chloride & C3 & WDR5-0103 \\
\hline C4 & ITF 2357 & C4 & AMI-1 (sodium salt) \\
\hline C5 & $\mathrm{PFI}-1$ & C5 & UNC1215 \\
\hline C6 & 5-Azacytidine & C6 & GSK 343 \\
\hline C7 & Decitabine & C7 & SIRT1/2 inhibitor IV \\
\hline C8 & $(+)-J \mathrm{Q} 1$ & $\mathrm{C} 8$ & I-CBP112 (hydrochloride) \\
\hline C9 & $(-)-J Q 1$ & C9 & UNC1999 \\
\hline C10 & BSI-201 & $\mathrm{C} 10$ & PFI-3 \\
\hline C11 & 1-Naphthoic acid & C11 & trans-Resveratrol \\
\hline D2 & Sodium 4-phenylbutyrate & D2 & 2,4-DPD \\
\hline D3 & $10 \times 1$ & D3 & DMOG \\
\hline D4 & Ml-2 (hydrochloride) & D4 & Trichostatin A \\
\hline D5 & MI-nc (hydrochloride) & D5 & CAY10398 \\
\hline D6 & Gemcitabine & D6 & RSC-133 \\
\hline D7 & Lomeguatrib & D7 & KD 5170 \\
\hline D8 & Octyl-a-ketoglutarate & D8 & CAY10433 \\
\hline D9 & Daminozide & D9 & Piceatannol \\
\hline D10 & GSK-J1 (sodium salt) & D10 & CAY10591 \\
\hline D11 & GSK-J2 (sodium salt) & D11 & EX-527 \\
\hline E2 & GSK-J4 (hydrochloride) & E2 & SAHA \\
\hline E3 & GSK-J5 (hydrochloride) & E3 & 2-PCPA (hydrochloride) \\
\hline E4 & $\mathrm{CPTH} 2$ & E4 & Nicotinamide \\
\hline
\end{tabular}


Table 1 Cayman chemical epigenetics screening library (Continued)

\begin{tabular}{llll}
\hline Plate $\mathbf{1}$ & & & Plate 2 \\
\cline { 1 - 2 } Well & Small molecule & Well & Small molecule \\
\hline E5 & Valproic acid (sodium salt) & E5 & BIX01294 \\
E6 & Tenovin-1 & E6 & N-Oxalylglycine \\
E7 & Tenovin-6 & E7 & Suramin (sodium salt) \\
E8 & Sodium butyrate & & \\
E9 & Anacardic acid & & \\
\hline
\end{tabular}

The identities of the chemicals and composition of the library, 94 chemicals distributed over two 96-well plates, are shown. The library (Cayman Chemical Item No. 11076, Batch No. 0455098) was received as $10 \mathrm{mM}$ stocks of each chemical in DMSO
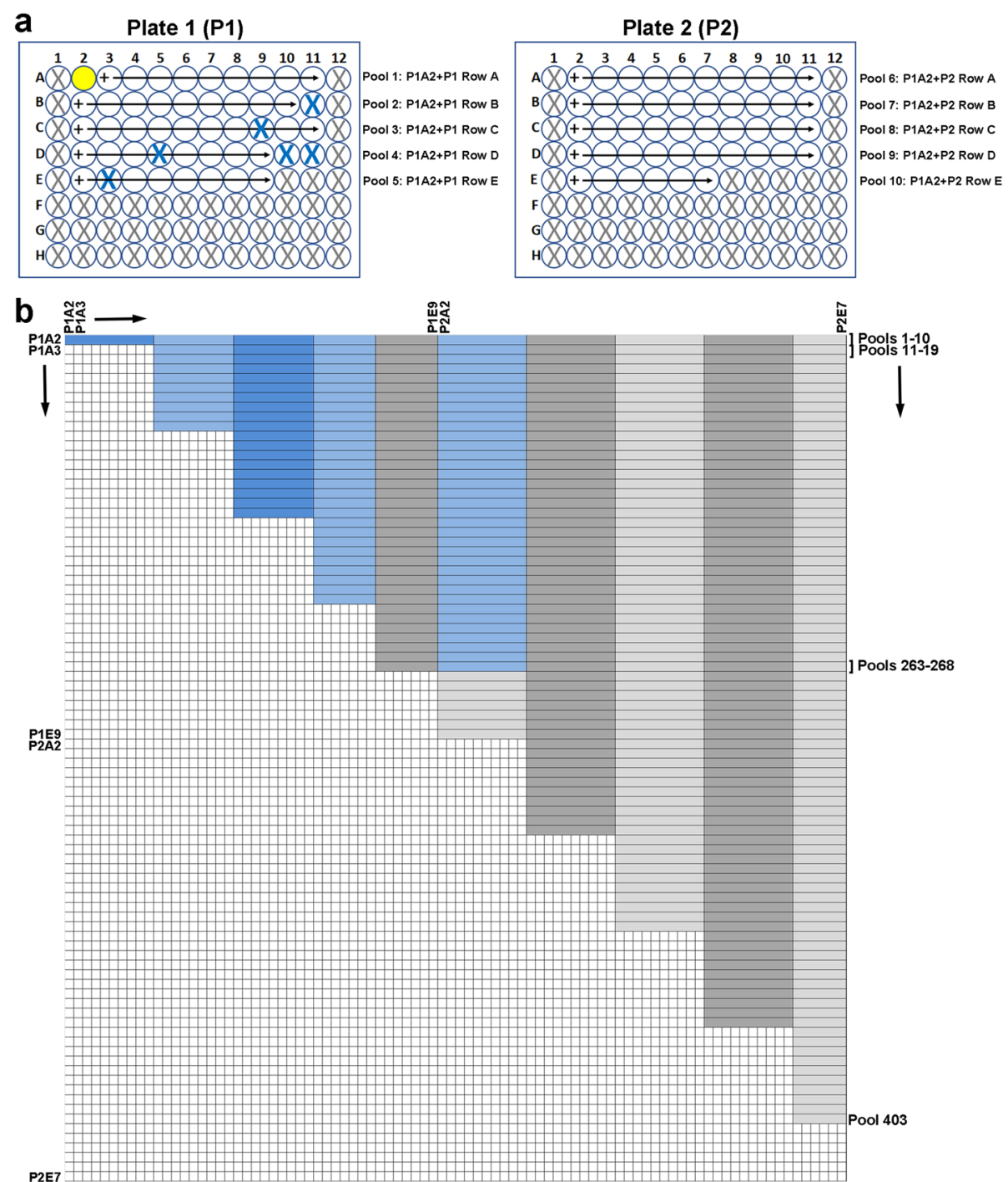

Fig. 1 Cayman Chemical Epigenetic Screening Library chemical-combination pooling scheme. a The library consists of 2 96-well plates (P1, P2). See Table 1 for the list of chemicals. Gray X's mark empty wells. Blue X's mark wells with chemicals not used in our screen: CAY10669, (-)-JQ1, MInc, GSK-J1, GSK-J2, and GSK-J5. Pooling process for plate 1 well A2 chemical (3-amino benzamide; yellow well) is illustrated. b Grid representation of the library chemicals and pools, in which each chemical (88 out of 94 total library chemicals) is represented along both the $x$ - and $y$-axes. CAY10669, (-)-JQ1, MI-nC, GSK-J1, GSK-J2, and GSK-J5 are removed from this grid representation. Shading (dark blue/light blue, dark gray/light gray) represents rows of chemicals in the library plates. Each horizontal, alternately shaded row represents a candidate chemical pool. Blue shading represents the 93 pools tested. Gray shading represents the remaining candidate pools (403 total pools on this grid) 
with 1\% DMSO in embryo medium (EM), or with $1 \%$ DMSO in EM as a vehicle control. Treatments were started at about 24 hpf and continued for 3 days, changing the treatment or vehicle control EM solution every $24 \mathrm{~h}$. Larvae were fixed in $4 \%$ paraformaldehyde (PFA) in PBS at $4 \mathrm{dpf}$ and stored at $4{ }^{\circ} \mathrm{C}$. To obtain tissue for genotyping, larval heads were removed with a scalpel at the level of the pectoral fins. Larval tails with trunk skeletal muscle remained intact and were maintained in $4 \%$ PFA until imaging was performed.

\section{Imaging and scoring muscle lesions}

Larvae fixed in 4\% PFA were rinsed in PBS with $0.01 \%$ Tween (PBSTw) prior to imaging. Animals were placed in PBSTw in a $60-\mathrm{mm}$ glass Petri dish. An Olympus SZX16 stereomicroscope with an attached Olympus DP72 camera was set up with one sheet of polarizing film over the trans-illumination base and another sheet of the film placed over the objective lens such that the two films were crossed [55]. For chemical pool screening, larvae were sorted under birefringence into muscle lesion positive or negative groups. The number of larvae in each group was recorded.

For quantitative birefringence measurements, we adapted an approach based on previously described methods $[47,55]$. In this approach, larvae were placed in a glass-bottom Petri dish in $2.5 \%$ methyl cellulose and oriented to maximize the brightness of the muscle tissue through the crossed polarizers. If necessary, pectoral fins and any remaining yolk tissue were removed so that the larvae could lie flat, to ensure consistent birefringence across the length of the trunk. Birefringence was imaged in individual animals as above. Images were acquired using the Olympus Cellsens Dimensions software. Exposure time was adjusted so that only a few saturated pixels were present. Adjusting exposure time in this way should control for any variation in brightness caused by larvae being at slightly different orientations. All other microscope and camera settings were kept constant. ImageJ was used to outline the trunk musculature, using the wand tool, and to calculate the average pixel intensity within the resulting selection. Average pixel intensity values for experimental conditions were normalized to wild-type control values. We see similar relative average pixel intensity values for control and mutant samples from experiment to experiment, indicating that our procedure is highly reproducible.

\section{Wholemount immunocytochemistry and phalloidin staining}

Whole-animal immunostaining was performed with the primary antibodies anti-dystrophin (1:100, Sigma D8043) and anti-beta dystroglycan (1:50, Novocastra NCL-bDG). The secondary antibody was either goat anti- mouse AlexaFluor-488 or goat anti-mouse AlexaFluor568 (1:200, Life Technologies/Molecular Probes). Four dpf larvae were fixed in $4 \%$ PFA in PBS for $4 \mathrm{~h}$ at room temperature and then washed into PBSTw. For antidystrophin staining, larvae were dehydrated through a methanol series and stored in $100 \%$ methanol at $-20^{\circ} \mathrm{C}$ overnight. They were then re-hydrated into PBSTw, washed $5 \times$ in PBSTw containing $1 \%$ DMSO, and blocked overnight at $4{ }^{\circ} \mathrm{C}$ in $2 \% \mathrm{BSA}, 2 \%$ heat inactivated normal goat serum, and $1 \%$ DMSO in PBSTw. The samples were then incubated with primary antibody diluted in the above block for $24 \mathrm{~h}$ at $4{ }^{\circ} \mathrm{C}$, washed with PBSTw $+1 \%$ DMSO, re-blocked for $6 \mathrm{~h}$ at room temperature, incubated with secondary antibody for $16 \mathrm{~h}$ at $4{ }^{\circ} \mathrm{C}$, washed with PBSTw $+1 \%$ DMSO, and stored in $4 \%$ PFA in PBS. For anti-beta dystroglycan staining, the fixed larvae were permeabilized with $2 \%$ Triton X-100 in PBS for $2 \mathrm{~h}$ at room temperature, then washed into PBSTw, and then blocked in 5\% BSA, 2\% heat-inactivated normal goat serum, $1 \%$ DMSO, $1 \%$ Triton $\mathrm{X}-100$, and $0.2 \%$ saponin in PBS overnight at $4{ }^{\circ} \mathrm{C}$. The samples were then processed as for the anti-dystrophin stained samples, except the block including Triton X-100 and saponin was used. To visualize actin in the muscle fibers of the betadystroglycan-stained animals, CF488A-conjugated phalloidin (1:50, Biotium Cat. \# 00042) was included with the secondary antibody. For confocal imaging, the heads and yolk were removed from representative larvae and the trunks/tails were mounted laterally in $80 \%$ glycerol containing $4 \%$ propyl gallate. Images were collected using a Leica TCS SP5 II confocal on a DMI6000 stand with a $20 \times$ NA 0.7 air objective.

\section{Immunoblotting}

Chemical treatments of embryos from $d m d+/-$ crosses were performed as above. At $4 \mathrm{dpf}$, heads were cut from anesthetized animals and were arrayed in 96-well plates for genotyping. The remaining trunk and tail portions of the animals were arrayed in separate 96-well plates, to which LDS sample buffer (Invitrogen NP0007) was added and stored at $-20^{\circ} \mathrm{C}$ until genotyping was completed. Trunk lysates were then pooled by genotype. Approximately, 0.75 animal equivalent per lane was separated on $12 \%$ NuPAGE Bis-Tris gels run in NuPAGE MES buffer (Invitrogen) and blotted. Blots were probed separately with anti-pan acetylated histone H4 (1:2000, rabbit polyclonal, Millipore Cat. \# 06-866) and anti-acetylated histone H4 K16 (1:2500, rabbit polyclonal, Millipore Cat. \# 07-329). Anti-actin was included with both anti-acetylated histone antibodies as a loading control (1:2000; mouse monoclonal Clone C4, MP Biomedical Cat. \# 69100). Infrared-dye labeled secondary antibodies (Rockland) were visualized using a Licor Odyssey infrared scanner. 


\section{Statistical analyses}

Statistical tests used are provided in the figure legends. Statistical analyses were performed and graphs were constructed using GraphPad Prism 8.

\section{Results}

\section{Epigenetic small-molecule library pooling approach}

In a previous study, we showed that the pan-HDACi TSA could improve the zebrafish $d m d$ muscle lesion phenotype [27]. We wanted to test whether we could identify new epigenetic small molecules, or epigenetic small molecule combinations, that improve the zebrafish $d m d$ muscle lesion phenotype. We thus decided to test the effects of small molecules from the commercially available Cayman Chemical Epigenetics Screening Library. The version of the library that we obtained contained 94 chemicals distributed over two 96-well plates (Table 1 and Fig. 1a). Prior to screening these chemicals on dmd mutant embryos, we performed dose and toxicity testing of each individual library compound on wild-type embryos (see the "Methods" section). Most chemicals showed little or no detrimental effect on embryo health and survival at a $1 \mu \mathrm{M}$ dose, so we selected $1 \mu \mathrm{M}$ to be the working dose for chemical screening for most of the library compounds. C646 and apicidin were toxic and caused embryo lethality at $1 \mu \mathrm{M}$ but not at $100 \mathrm{nM}$, so these compounds were used at $100 \mathrm{nM}$. CAY10669 exhibited strong toxicity and embryonic lethality by $3 \mathrm{dpf}$ (days post-fertilization) and was removed from further screening. GSK-J1 is reported to be non-cell permeable and was removed from further screening (Fig. 1a). We also removed library chemicals that are designated as negative control compounds from further screening ((-)-JQ1, MI-nc, GSK-J2, and GSK-J5; Table 1; Fig. 1a).

Previous large-scale chemical screens in $d m d$ mutant zebrafish either tested individual chemicals or tested chemical pools by subdividing libraries into unique chemical pools containing eight compounds each [45, $48,49]$. In contrast to these previous screens, we wanted a strategy that would test each compound in combination with every other library compound. In order to efficiently test combinations of epigenetic small molecules in the library, we designed a grid system consisting of a set of 403 different chemical pools (Fig. 1a, b). In this system, compound A2 of plate 1 is pooled with the remaining compounds of row A of plate 1 for combination pool 1 (Fig. 1a, b). Compound A2 is then pooled with compounds from plate 1 row B (pool 2), plate 1 row $C$ (pool 3), and the remaining rows from the library for pools 4-10 (Fig. 1a, b). Compound A3 of plate 1 is then combined with compounds of plate 1 row $B$ for pool 11, and so on (Fig. 1a, b). In this grid system, each compound would be tested in combination with each of the other library chemicals in at least 1 pool (Fig. 1a, b).
To determine if screening pools of epigenetic small molecules was sensitive enough to identify compounds that could improve the zebrafish $d m d$ muscle phenotype, we tested the pool of 10 chemicals from plate 2 row $\mathrm{D}$, which contains TSA (Table 1). Animals from $d m d+/-$ crosses were treated from 1-4 days and then scored for muscle birefringence (as in [27] and illustrated in Fig. 2). Treatment with the pool of chemicals from plate 2 row D significantly decreased the number of affected animals exhibiting abnormal muscle birefringence (Fig. 3). These results indicate that a pool of compounds containing a known beneficial epigenetic small molecule, TSA, can improve the zebrafish $d m d$ muscle phenotype. These results suggest that the epigenetic chemical-pooling design of our screen is sensitive enough to pick up positive hits of small molecules that improve the zebrafish $d m d$ muscle phenotype.

\section{Pilot screen of epigenetic small-molecule combination pools}

We then proceeded to test epigenetic small molecule pools from our chemical-combination grid system (Fig. 1). We tested 93 of the 403 possible chemical pool combinations (Figs. $1 \mathrm{~b}$ and $4 \mathrm{a}$ ). Animals from $d m d+/-$ crosses were treated from 1-4 days and then scored for muscle birefringence. Our approach for assessing the dystrophic phenotype using birefringence in our pilot library screen followed the approach used in previous zebrafish $d m d$ mutant chemical screens [45, 48, 49], in which control and drug-treated animals are scored as "affected," or showing a clear $d m d$ mutant muscle lesion phenotype by birefringence, or "unaffected," or showing largely normal birefringence. Each pool was tested in duplicate, with 25 animals per well for each replicate. We determined the average percentage of affected animals from each chemical pool, and from each DMSO control tested, and we plotted the results as a heat map on our combination pool grid (Fig. 4a). This heat map grid reveals that the majority of pools tested that included plate 2 row A reduce the percentage of animals affected (column 5 in Fig. 4a). To test whether the plate 2 row A pools are significantly improving the percentage of animals affected, we plotted the combined results from all pools containing each plate row compared with the combined results from DMSO control treatments (Fig. 4b). This analysis shows that treatment pools using plate 2 row A led to a significant reduction in the percentage of affected animals relative to the DMSO controls (Fig. $4 \mathrm{~b})$. On average, treatment with chemical pools that included plate 2 row A resulted in only about $10 \%$ affected animals, compared to the $25 \%$ affected average observed in the DMSO controls (Fig. 4b). Figure 4c and d shows an example of the improved birefringence from animals treated with a representative pool from the plate 2 row 


Collect eggs from
$\begin{gathered}\text { Tmd+/- } \mathbf{X} \mathbf{d m d + /} \\ \text { with epigenetic }\end{gathered}$

A pools (pool 135 in Fig. 1b; pilot screen pool \#59), compared to sibling DMSO controls. While these results do not rule out beneficial effects of compounds or treatment pools from our screen that did not include plate 2 row $\mathrm{A}$, these results do highlight that compounds within plate 2 row A are having significant beneficial effects on the zebrafish $d m d$ muscle lesion phenotype.

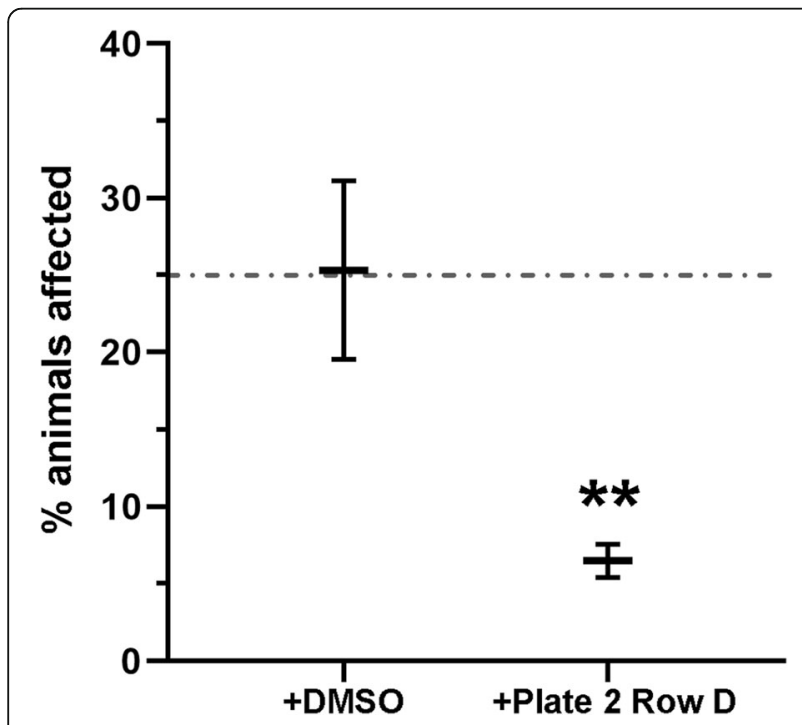

Fig. 3 Plate 2 row D chemical pool, which includes TSA, significantly reduces the frequency of zebrafish $d m d$ mutant animals exhibiting abnormal muscle birefringence. Control treatment is 1\% DMSO. The dashed line represents the expected $25 \%$ affected animals. See Table 1 for the 10 chemicals in plate 2 row D. Each chemical was used at $1 \mu \mathrm{M}$. For each treatment condition, $n=3$ replicates, with 23-27 embryos in each replicate. Error bars represent standard error. Significance was determined using a Mantel-Haenszel test. ${ }^{* *} p<$ 0.004 compared to DMSO control
Analysis of plate 2 row A small molecules identifies a beneficial combination of oxamflatin and salermide While useful for screening purposes, the approach of scoring animals as "affected" versus "unaffected" may only provide an approximate measure of the effects of small molecules on the dmd mutant phenotype, as animals scored as "affected" could still be improved over typical $d m d$ mutants, and "unaffected" larvae could still have dystrophic lesions but are substantially less severe than is typical for $d m d$ mutants. To determine which compounds mediate the effect of plate 2 row A, we wanted an approach that would allow us to more quantitatively assess the effects of small molecules on zebrafish dmd mutant birefringence. Adapting previously described methods $[47,55]$, we used gray value measurements to quantitate the brightness level of the muscle birefringence of each animal (Fig. 5a). For each animal, we outlined a unilateral area of trunk muscle birefringence and measured the average pixel brightness within that area (Fig. 5a). Zebrafish $d m d$ mutant animals show reduced birefringence, as measured by lower pixel intensity, compared to their control siblings (Fig. 5c, e). As a test of this approach, we confirmed that TSA treatment significantly improved the average pixel brightness of dmd mutant birefringence (Fig. $5 \mathrm{~d}, \mathrm{e}$ ).

We, next tested each compound from plate 2 row A to see if any of the individual chemicals could improve the $d m d$ birefringence phenotype. Animals from $d m d+/-$ crosses were treated from 1-4 days and then scored for muscle birefringence. When tested individually, none of the compounds showed a significant effect on the DMSO-treated $d m d$ muscle birefringence brightness (Fig. 5f). These results indicate that a combination of chemicals from plate 2 row A is needed to improve the $d m d$ mutant birefringence phenotype. 

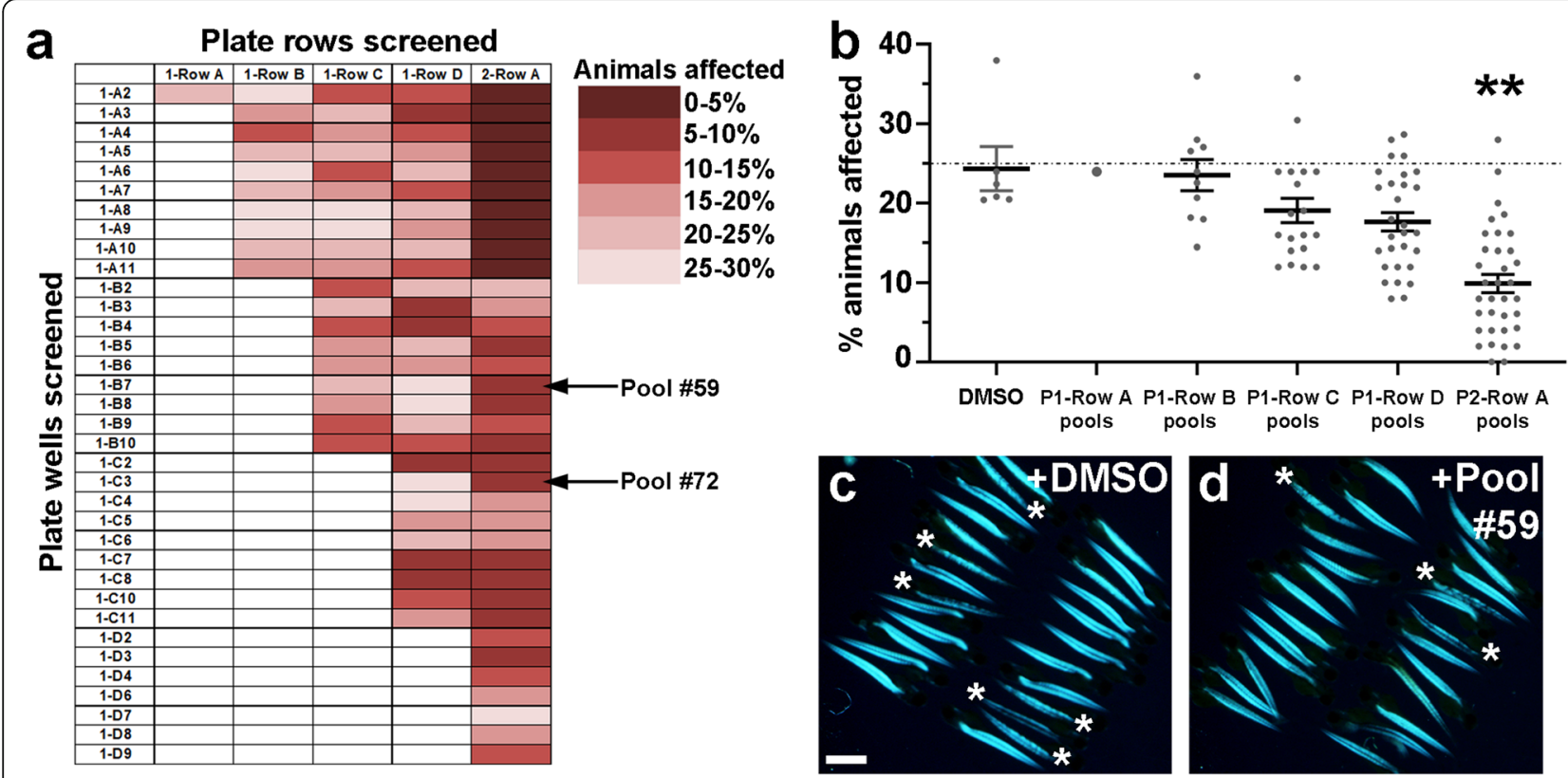

Fig. 4 Pilot screen identifies the ability of plate 2 row A chemical pools to lower the frequency of affected dmd animals. (a) Heat map representation of the average percentage of affected animals observed from each of the 93 pools tested. Individual chemical wells tested were from plate 1 (left column, plate number and well number shown). Library rows tested are rows A-D from plate 1 and row A from plate 2. See Table 1 for the chemicals in each well and row. Arrows point to pilot screen pool \#59 (pool 135 in Fig. 1b), shown in (d), and pilot screen pool \#72 (pool 173 in Fig. 1b), analyzed in Fig. 5 below. (b) Graph of combined average percentages of affected animals from all tested pools that included each plate row. Control treatments are 1\% DMSO. The dashed line represents the average of the control DMSO treatments (25\%). Each dot represents a tested drug pool's average of affected animals. Error bars represent standard error. Significance was determined using a one-way ANOVA test comparing each chemical pool group to the DMSO control group with Dunnett's correction for multiple comparisons. ${ }^{* *} p=0.0021$ compared to DMSO control. (c, d) Example of birefringence images of 4 dpf larvae from a replicate of (c) DMSO control and (d) pool \#59 (plate 2 row A + UNC0638) treatments. DMSO control animals are siblings of pool \#59 animals. Asterisks mark affected animals. Scale bar $=1 \mathrm{~mm}$

To identify the compounds required for the effects of plate 2 row $\mathrm{A}$, we next decided to remove individual chemicals from the drug pool. We chose to test pilot screen pool \#72 (pool 173 in Fig. 1b; plate 2 row A + plate 1 well $\mathrm{C} 3$; 11 total chemicals), because this pool represented an average effect from the plate 2 row A pools (Fig. 4a, b). As above, animals from $d m d+/-$ crosses were treated from 1-4 days and then scored for muscle birefringence. Treatment with the full 11 chemicals from pool \#72 showed significant improvement of the $d m d$ mutant birefringence using our quantitative analysis (Fig. 6a). However, the removal of four different chemicals (chaetocin, oxamflatin, salermide, and delphinidin chloride) each inhibited the ability of pool \#72 to improve $d m d$ birefringence (Fig. 6a), indicating that these four compounds are involved in producing the $d m d$ rescue effect of pool \#72.

We next focused on the roles of chaetocin, oxamflatin, and salermide. We decided not to further test the role of delphinidin chloride primarily because it is not a component of the plate 2 row A compounds on which we decided to focus (delphinidin chloride is the plate 1 well C3 compound in pool \#72) and also because it did not exhibit beneficial activity on its own (data not shown). To further test the roles of chaetocin, oxamflatin, and salermide in improving $d m d$ muscle birefringence, we tested these three compounds together and in each pairwise combination (Fig. 6b). The combination of oxamflatin and salermide significantly improved $d m d$ mutant muscle birefringence, similarly to pool \#72, whereas the other pair-wise combinations and the three-chemical combination did not (Fig. 6b). Figure 6c-f shows examples of the improved birefringence from animals treated with pool \#72 (Fig. 6e) and the combination of oxamflatin+salermide (Fig. 6f). While these findings do not rule out possible contributions of delphinidin chloride and chaetocin, these results show that the combination of oxamflatin and salermide can mediate the beneficial effects of pool \#72 in improving dmd mutant muscle birefringence.

\section{An independent laboratory test validates the beneficial effects of oxamflatin+salermide}

To validate these effects of oxamflatin and salermide, we performed treatments from $1 \mathrm{dpf}-4 \mathrm{dpf}$ at an independent site, in the laboratory of Dr. Henry. We observed 


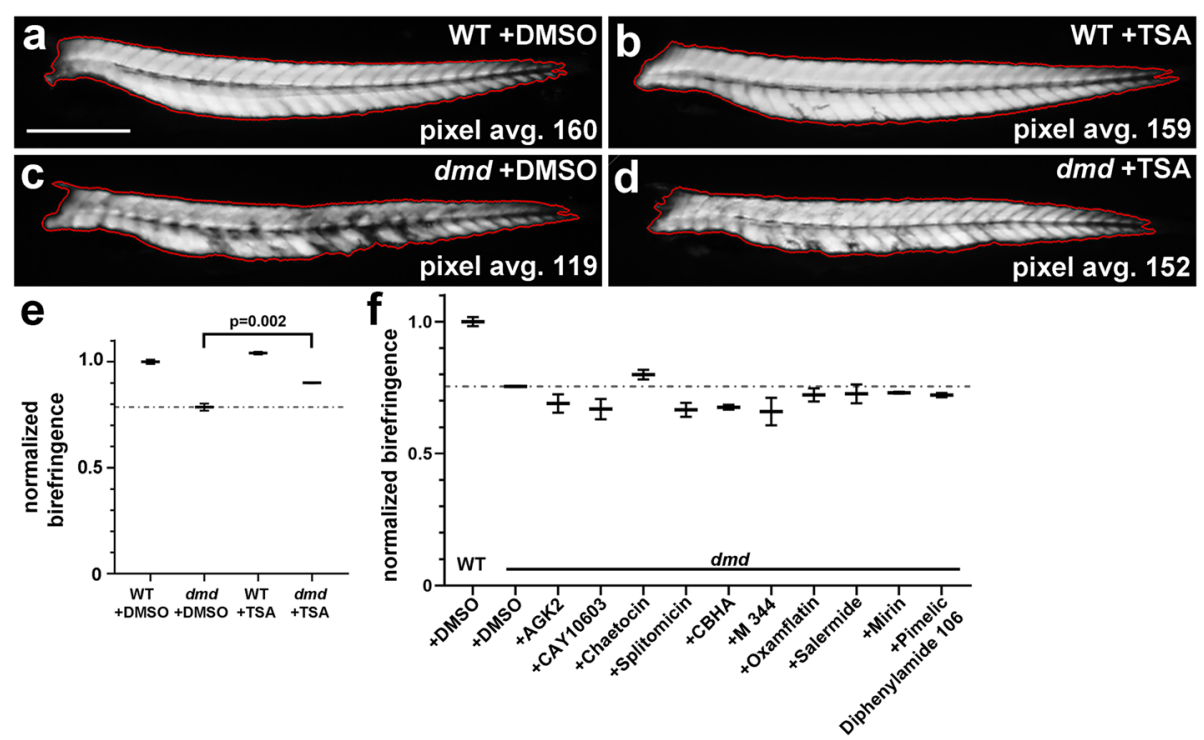

Fig. 5 Individual compounds from plate 2 row A do not significantly improve zebrafish dmd mutant muscle birefringence. a-d Four dpf zebrafish trunk muscle, with trunk skeletal muscle birefringence outlined in red. Lateral views, anterior to the left. Average pixel brightness values are shown for (a) wild-type (WT) + DMSO, (b) WT + TSA, (c) dmd + DMSO, and (d) dmd + TSA. Scale bar = $500 \mu m$. e Graph of normalized birefringence pixel intensities for $200 \mathrm{nM}$ TSA treatments vs DMSO controls. $n=3$ biological replicates for each treatment. Plot shows the average normalized pixel intensity for the 3 replicates for each treatment (4-9 genotyped animals per replicate). The dashed line represents the average normalized pixel intensity for all of the DMSO-treated $d m d$ animals $(n=17)$. Error bars represent standard error. $p$ value determined by Student's $t$ test. (f) Graph of normalized birefringence pixel intensities for treatments of zebrafish dmd mutants with the 10 individual chemicals from plate 2 row A. Control treatment is $1 \%$ DMSO. All chemicals were tested at $1 \mu \mathrm{M}$. For each treatment condition, $n=3$ replicates, with 2-9 dmd embryos in each replicate. Plot shows the normalized birefringence pixel intensity for the 3 replicates for each treatment. The dashed line represents the average normalized pixel intensity for all of the DMSO-treated $d m d$ animals $(n=15)$. Error bars represent standard error. Significance was determined using a one-way ANOVA test comparing each treatment group to the dmd DMSO control group with Dunnett's correction for multiple comparisons. $p>0.2$ for all individual chemicals compared to dmd DMSO control

similar improvement of zebrafish $d m d$ mutant muscle birefringence with oxamflatin+salermide treatments performed in the Henry Lab (Fig. 6g) as we observed with treatments performed in the Maves Lab (Fig. 6b). Dr. Henry's lab also validated that TSA-improved zebrafish dmd mutant birefringence (Fig. 6h). By demonstrating reproducibility, these independent tests are a strong validation of the benefits of these small molecules, and in particular the novel oxamflatin+salermide combination, for $d m d$ mutant zebrafish.

\section{Oxamflatin and salermide have dose-dependent effects, independent of dystrophin expression}

To further address the effects of oxamflatin and salermide, we performed a dose-response analysis. We tested concentrations of oxamflatin and salermide, from $0.5 \mu \mathrm{M}$ to $4 \mu \mathrm{M}$, both individually and in combination (Fig. 7a). As above, animals from $d m d+/-$ crosses were treated from 1-4 days and then scored for muscle birefringence. We found that oxamflatin and salermide in combination at doses lower than $1 \mu \mathrm{M}$ do not improve the $d m d$ mutant birefringence phenotype, while a combination dose of $2 \mu \mathrm{M}$ oxamflatin+salermide improves birefringence to a degree similar to that seen with the $1 \mu \mathrm{M}$ combination dose (Fig. 7a). Four micromolar and $2 \mu \mathrm{M}$ doses of oxamflatin alone improve the birefringence phenotype, but not as well as the $1 \mu \mathrm{M}$ and $2 \mu \mathrm{M}$ combination doses (Fig. 7a). Salermide alone did not improve muscle birefringence at any dose tested. We also found that a combination dose of $4 \mu \mathrm{M}$ oxamflatin+salermide was toxic and caused lethality by $4 \mathrm{dpf}$ (data not shown). These results reveal that, while oxamflatin alone can have beneficial effects, the combination of oxamflatin+salermide appears more effective at improving the dmd mutant birefringence phenotype.

We next asked whether oxamflatin and salermide are acting by upregulating dystrophin expression. Dystrophin expression is lost in $d m d^{\text {ta222a }}$ mutants (Fig. 7d) [39]. One to four dpf treatments with $1 \mu \mathrm{M}$ oxamflatin+ salermide do not cause any noticeable upregulation of dystrophin expression (Fig. 7e). We also examined the expression of $\beta$-dystroglycan ( $\beta D G$ ), a marker of the DAPC at the vertical myosepta in the zebrafish trunk $[39,56]$. Previous studies have shown that $\beta D G$ expression is largely still intact at the myosepta in $d m d$ mutants (Fig. 7h) [39, 57], although see [58]. BDG-labeled 


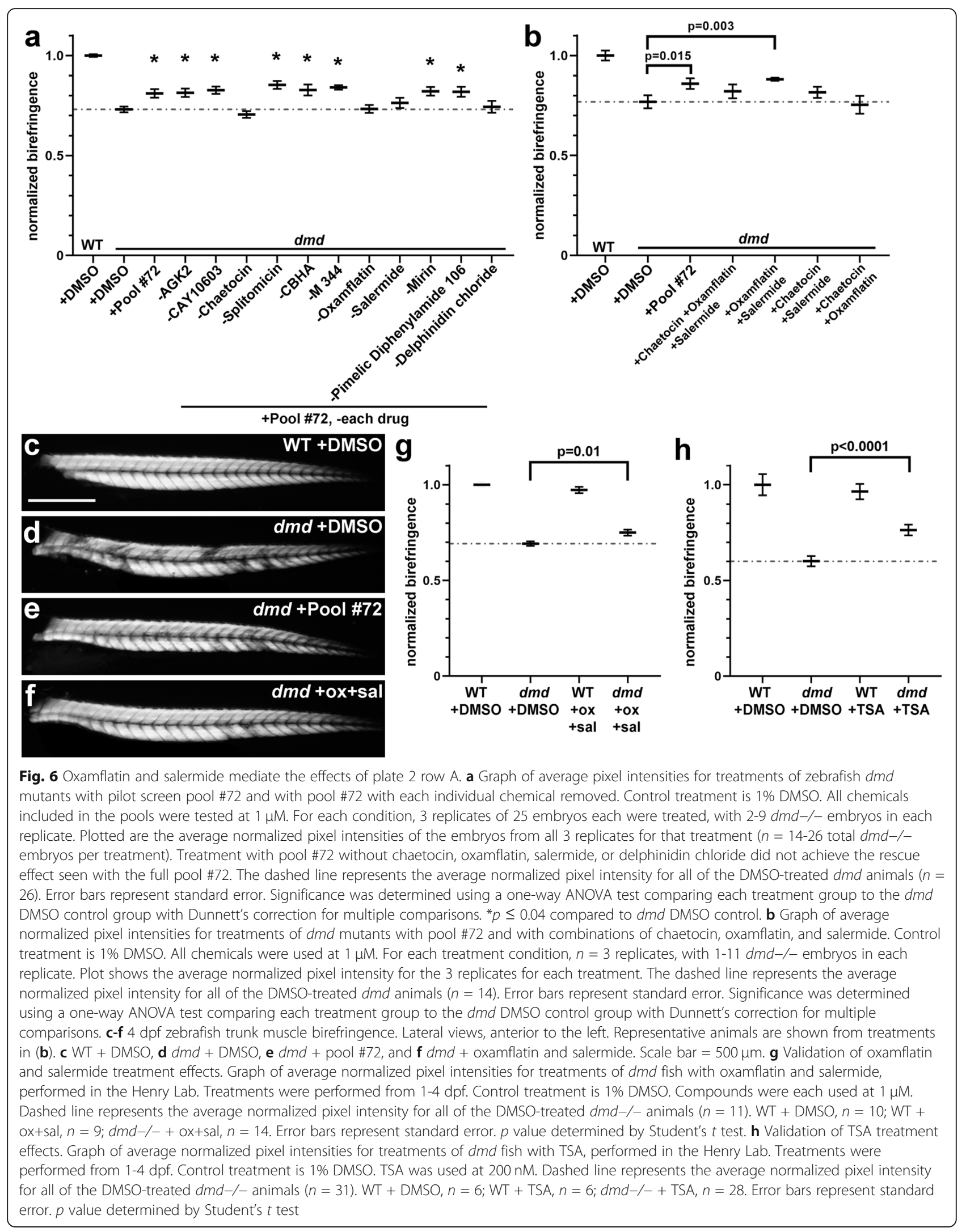



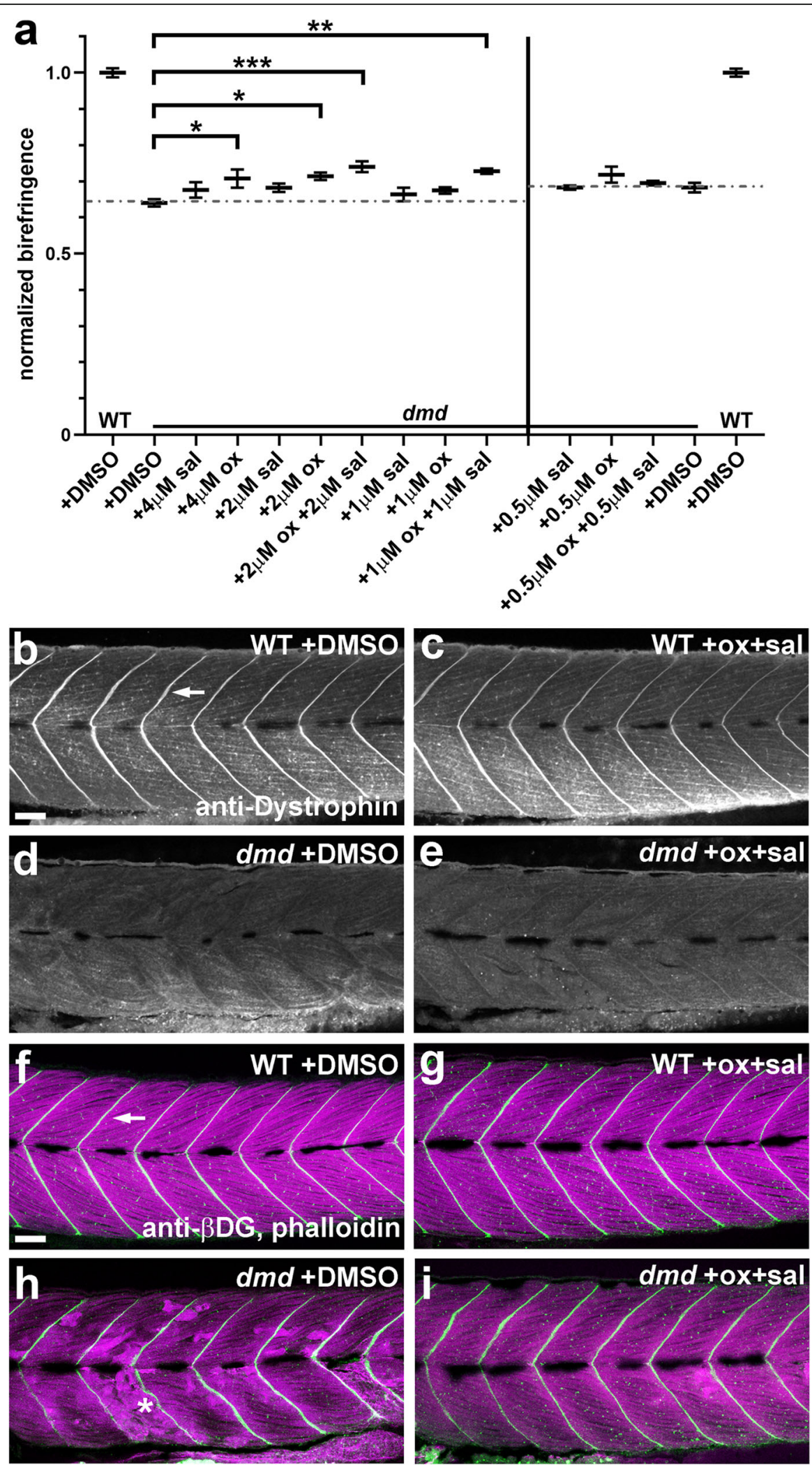

Fig. 7 (See legend on next page.) 
(See figure on previous page.)

Fig. 7 Oxamflatin and salermide have dose-dependent effects, independent of dystrophin expression. a Graph of average normalized pixel intensities for treatments of $d m d$ mutants with doses of oxamflatin and salermide. Control treatment is $1 \%$ DMSO. Chemicals were used between $0.5 \mu \mathrm{M}$ and $4 \mu \mathrm{M}$, over two separate experiments. For each treatment condition, $n=4$ replicates, with 2-11 dmd-/- embryos in each replicate. Plot shows the average normalized pixel intensity for each of the 4 replicate pools for each treatment. The vertical line separates the treatment conditions from the two experiments, each of which has its own WT + DMSO and dmd + DMSO controls. The dashed lines represent the average normalized pixel intensity for all of the DMSO-treated $d m d$ animals ( $n=26$ and $n=30)$. Error bars represent standard error. Significance was determined using a one-way ANOVA test comparing each treatment group to the dmd DMSO control group with Dunnett's correction for multiple comparisons. ${ }^{*} p \leq 0.029,{ }^{* *} p=0.0029,{ }^{* * *} p=0.0007$ compared to dmd DMSO control. b-e Confocal images of anti-dystrophin staining in the trunk musculature of $4 \mathrm{dpf} \mathbf{b} \mathrm{WT}+\mathrm{DMSO}, \mathbf{c} W T+$ oxamflatin and salermide, $\mathbf{d} d m d+\mathrm{DMSO}$, and $\mathbf{e} d m d+$ oxamflatin and salermide larvae. Lateral views, anterior to the left. Arrow points to dystrophin expression in the vertical myoseptum. All $d m d+/+$ animals showed normal dystrophin expression (WT + DMSO, $n=16$; WT + ox+sal, $n=14)$ and all $d m d-/-$ animals lacked detectable dystrophin expression (dmd-/ -+ DMSO, $n=23$; $d m d-/-+$ ox + sal, $n=18$ ). Scale bar $=50 \mu \mathrm{m}$. $\mathbf{f - i}$ Confocal images of anti- $\beta$-dystroglycan (BDG) and phalloidin staining in the trunk musculature of $4 \mathrm{dpf} \mathbf{f} \mathrm{WT}+\mathrm{DMSO}, \mathbf{g} \mathrm{WT}+$ oxamflatin and salermide, $\mathbf{h} d m d+\mathrm{DMSO}, \mathbf{i} d m d+$ oxamflatin and salermide. Lateral views, anterior to the left. Arrow points to $\beta D G$ expression (white) in the vertical myoseptum. Phalloidin staining of filamentous actin (magenta) shows the disrupted muscle structure in dmd mutants $\left(^{*}\right.$ in $\left.\mathbf{h}\right)$. All wild type animals $(+/+$ and $+/-)$ showed normal $\beta$-dystroglycan expression (WT + DMSO, $n=27 ; \mathrm{WT}+$ ox+sal, $n=26)$, and $d m d-/-$ animals showed largely maintained $\beta$-dystroglycan expression $(d m d-/-+\mathrm{DMSO}, n=9 ; d m d$ $-/-+$ ox+sal, $n=14)$. Scale bar $=50 \mu \mathrm{m}$

myosepta, and overall muscle fiber structure, appear improved by $1 \mu \mathrm{M}$ oxamflatin+salermide treatments in $d m d$ mutants, and we do not observe any obvious upregulation of $\beta D G$ or actin filaments (Fig. 7i). These results suggest that oxamflatin+salermide are acting independently of dystrophin upregulation and further demonstrate the improved muscle structure of $d m d$ mutants caused by oxamflatin+salermide treatments.

\section{Oxamflatin and salermide together increase histone acetylation}

We next wanted to address the mechanisms by which oxamflatin and salermide might be working to improve the dmd mutant phenotype. Oxamflatin is an HDACi that inhibits class I and II HDACs [59,60]. Salermide is a class III HDACi that inhibits the NAD + -dependent deacetylases SIRT1 and SIRT2 [61]. Oxamflatin and
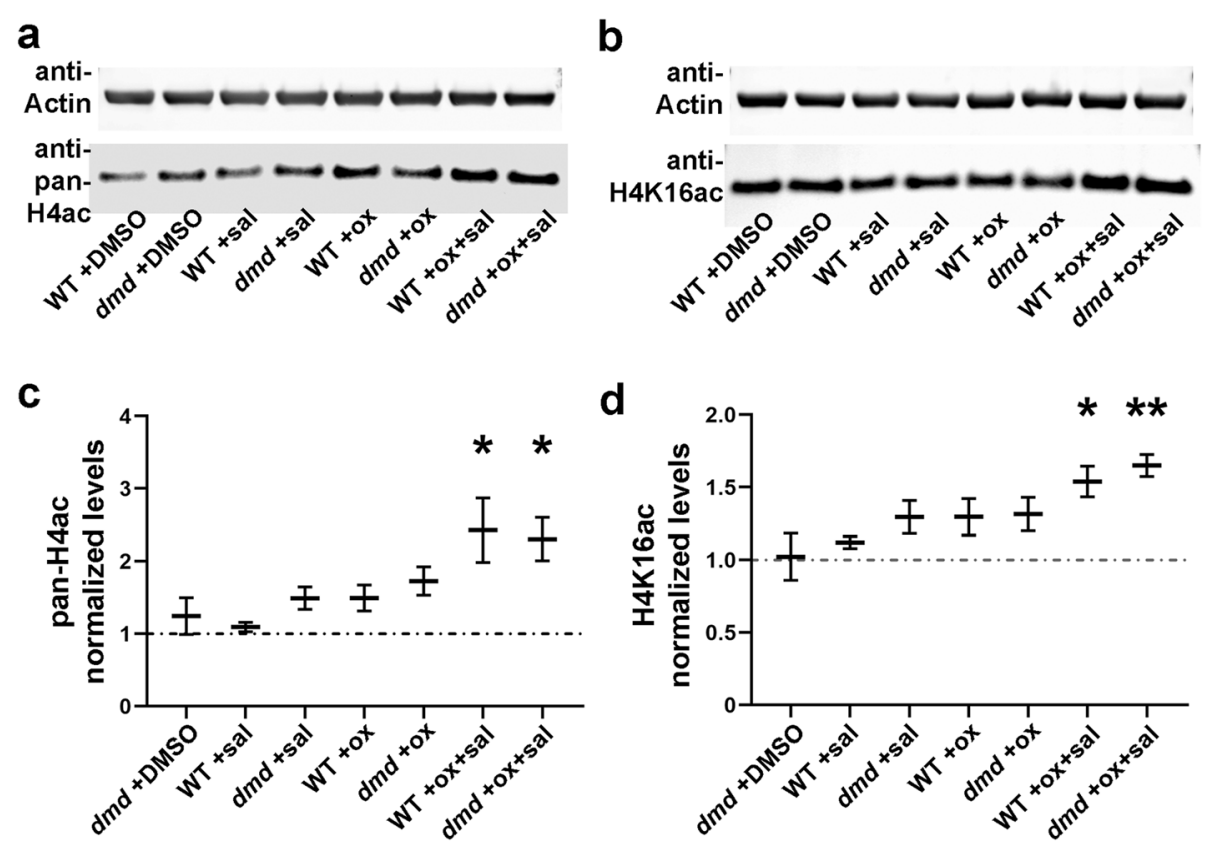

Fig. 8 Oxamflatin and salermide together increase histone acetylation. a-b Representative Western blots of (a) histone $\mathrm{H} 4$ acetylated at lysines 5 , 8, 12, and 16 (pan-H4ac) and (b) histone $\mathrm{H} 4$ acetylated specifically at lysine 16 (H4K16ac). Lysates are from genotyped 4 dpf larvae. c-d Quantification of Western blot analyses of levels of (c) pan-H4ac and (d) H4K16ac. Lysates were prepared from 4 replicate pools of animals for each treatment condition, with $n=4-10$ animals per genotype per replicate. For each sample, pan-H4ac or H4K16ac signal was normalized to actin as a loading control. This value was then normalized to the WT + DMSO value within each set of samples. The plots show the average normalized signals of the 4 replicates. Error bars represent standard error. Significance was determined using a Student's $t$ test with Welch's correction for unequal sample variances. In $(\mathbf{c}),{ }^{*} p<0.05$. In $(\mathbf{d}),{ }^{*} p=0.015,{ }^{* *} p=0.003$ compared to WT + DMSO control. Pan-H4ac levels are increased an average of $2.4 \times$ in oxamflatin+salermide-treated animals, and H4K16ac levels are increased an average of 1.6x 
salermide have each been shown to cause increased histone acetylation in mammalian cell culture models [60, 61]. Salermide has also been shown to cause increased acetylation of lysine 16 of histone H4 (H4K16ac), a histone modification target of SIRT1 and SIRT2 [61-63]. We therefore asked whether oxamflatin and salermide treatments cause increased histone acetylation in $d m d$ mutant zebrafish. We treated animals from $d m d+/-$ crosses from 1-4 days and then collected animals at 4 $\mathrm{dpf}$ for protein lysates. Western analysis showed that treatments of $1 \mu \mathrm{M}$ oxamflatin+salermide caused increased levels of pan-acetyl histone H4 and H4K16ac in both control and $d m d$ mutant animals (Fig. 8a-d). However, $1 \mu \mathrm{M}$ treatments of either oxamflatin or salermide did not increase levels of these histone marks (Fig. 8a-d). These results show that these small molecules are indeed acting as HDACi in zebrafish larvae and begin to reveal a possible mechanism for the activity of this chemical combination.

\section{Discussion}

In this study, we performed a screen of epigeneticmodifying small molecules to identify compounds that prevented muscle degeneration in the zebrafish model of DMD. We developed a novel chemical-pooling approach to screen combinations of epigenetic small molecules, and we performed a pilot screen of 93 novel epigenetic small molecule pools. While our pilot screen identified several new candidate small molecule pools that have beneficial effects on zebrafish dmd mutants, our chemical-pooling strategy and subsequent analysis highlighted the activity of compounds from plate 2 row A (Fig. 4). We were able to resolve a novel small molecule combination, oxamflatin and salermide that significantly reduced the number of $d m d$ animals with muscle lesions when compared to DMSO-treated controls. Our results provide support for further investigations of oxamflatin and salermide as potential therapeutic compounds for DMD. Our results also support additional screening of epigenetic small molecules for their beneficial effects in $d m d$ mutant zebrafish.

Several previous large-scale chemical screens have demonstrated the potential of $d m d$ mutant zebrafish for identifying new therapeutic compounds and targets [43, $45,48,49]$. These studies, and our work here, took advantage of two key approaches, the birefringence assay and drug pooling, to efficiently screen large numbers of compounds. Assessing the percentage of "affected" animals in large-scale $d m d$ mutant zebrafish chemical screens is efficient because the numbers of animals involved preclude performing genotyping. While there is potential for false negatives from these chemical screens, validation studies that incorporate animal genotyping and quantitative birefringence analysis, such as we use here with oxamflatin and salermide treatments, can alleviate concerns of false positives from chemical screens.

In contrast to previous screens that subdivided chemical libraries into unique chemical pools containing eight compounds each [45, 48], we wanted a strategy that would test each compound in combination with every other library compound. We designed a grid system in which each compound would be tested in combination with each of the other library compounds in at least 1 chemical pool (Fig. 1). By taking this novel drug pooling approach, we wanted to identify epigenetic small molecule combinations that are beneficial for $d m d$ mutant zebrafish, and we identified the new combination of oxamflatin and salermide. While our particular drug pooling approach may not be easily feasible for larger library screens, taking similar systematic approaches to testing combinations of chemical library compounds could be fruitful for DMD and other diseases.

Based on results from our pilot screen, we focused our studies here on oxamflatin and salermide. Oxamflatin is an HDACi that inhibits class I and II HDACs and is chemically similar to TSA $[59,60]$. Salermide is a class III HDACi that inhibits the NAD + -dependent deacetylases SIRT1 and SIRT2 [61], and so represents a new class of HDACi for DMD. In mammalian cell culture models, oxamflatin and salermide have each been shown to cause increased histone acetylation [60,61]. Oxamflatin has been shown to inhibit cell proliferation and induce cell shape changes in cancer cell lines $[60,64,65]$. Salermide has been shown to inhibit cell proliferation and induce apoptosis in cancer cell lines, acting through reactivation of pro-apoptotic genes that are repressed by SIRT1-mediated H4K16ac deacetylation [61, 66, 67]. The H4K16ac mark is highly conserved, inhibits chromatin compaction, and has been associated with cell cycle progression, dosage compensation, cancer, and lifespan [68]. Our finding that oxamflatin+salermide caused increased levels of pan-acetyl histone $\mathrm{H} 4$ and H4K16ac in both wild-type and $d m d$ mutant zebrafish (Fig. 8) shows that these HDACi compounds are having the expected biochemical effects on zebrafish larvae and also reveals that the combination of oxamflatin+salermide may be synergizing to activate specific histone marks.

In previous studies, overexpression of SIRT1 has been shown to ameliorate DMD pathophysiology in $m d x$ mice [69], while salermide inhibition of SIRT1 has been shown to protect muscle cells against oculopharyngeal muscular dystrophy in Caenorhabditis elegans [70]. SIRT1 and SIRT2 can have opposing effects in promoting angiogenesis and in providing neuroprotective effects in neurodegenerative disease models [71, 72]. Future studies are needed to determine whether oxamflatin and salermide are working additively or synergistically to provide skeletal muscle benefits in zebrafish $d m d$ 
mutants and whether they are acting through additional mechanisms that are independent of histone acetylation.

While many DMD small molecule therapies are being developed and entering clinical trials, some have difficulties showing efficacy in patients, possibly due to inadequate preclinical evaluation or targeting mechanisms too late in disease progression $[7,16,19]$. Underscoring the significance of this issue, a recent study was unable to replicate the benefits of the serotonin pathway modulator fluoxetine for $d m d \mathrm{mu}-$ tant zebrafish [73]. Here, we take advantage of the zebrafish model for DMD drug discovery and initial mechanistic validation. We also validated the effects of the epigenetic small molecules TSA and oxamflatin and salermide on $d m d$ mutant zebrafish in an independent laboratory (Fig. 6). A critical next step will be to validate the effects of oxamflatin and salermide in mammalian DMD models, such as $m d x$ mice, the Dmd rat, or human-induced pluripotent stem cell models of DMD [74-76]. We predict that small molecule validation in independent laboratories and in multiple model systems will increase the potential for future translation of epigenetic small molecule therapies and other pharmacological approaches for DMD.

The development of significantly beneficial therapies for DMD will likely involve the simultaneous use of a combination of therapies that target dystrophin and/or downstream pathological mechanisms [19, 24]. Epigenetic small molecules may represent a promising component of a DMD combination therapy. Epigenetic small molecules are outstanding candidates for a DMD pharmacological therapy for many reasons. Certain epigenetic small molecules have been shown to benefit DMD and other neuromuscular disorders [25, 77-80]. Several HDACi, including SAHA, and other epigenetic small molecules are already FDA-approved for use in cancers [81-83]. While some epigenetic small molecules have off-target or solubility issues, there is potential for optimizing dosing or using these chemicals as lead compounds for further analyses [81, 83]. In addition, there are growing numbers of examples showing that epigenetic small molecules synergize with each other or with other compounds to target different diseases, including cancer and heart disease [59, 81-85]. In our work here, we show an example of a combination of two epigenetic small molecules that are beneficial for $d m d$ mutant zebrafish. Because of the advantages of the zebrafish animal model, we expect that $d m d$ mutant zebrafish will be an increasingly important model for investigating combination therapies for DMD.

\section{Conclusions}

- We developed a new chemical-pooling approach for screening combinations of small molecules in $d m d$ mutant zebrafish.
- We identified a novel combination of epigenetic compounds, oxamflatin and salermide, that together improve skeletal muscle structural defects in $d m d$ mutant zebrafish.

- We have taken an important step in small molecule validation for DMD by demonstrating that oxamflatin and salermide show beneficial effects on zebrafish $d m d$ mutants in two independent laboratories.

\section{Abbreviations}

BDG: $\beta$-dystroglycan; DAPC: Dystrophin-associated protein complex; DMD: Duchenne muscular dystrophy/dystrophin; DMSO: Dimethyl sulfoxide; dpf: Days post-fertilization; EM: Embryo medium; H4ac: Pan-acetylation of histone H4; H4K16ac: Acetylation of lysine 16 of histone H4; HDACi: Histone deacetylase inhibitors; hpf: Hours post-fertilization; PFA: Paraformaldehyde; TSA: Trichostatin A; WT: Wild-type

\section{Acknowledgements}

We would like to thank the SCRI Office of Animal Care for caring for the zebrafish. We thank Kimia Imani, Max Urbanek, and Ermias Yohannes for technical assistance.

\section{Authors' contributions}

GHFIII performed experiments, conducted data analyses, and prepared figures. MM, AG, TP, and EUP performed chemical screening and data analysis. EK and $\mathrm{CH}$ performed validation tests. GHFIII and SS developed the quantitative birefringence assay. SS provided technical assistance. LM conceived the project, obtained funding, and assisted and consulted on all experiments, data analysis, and figure preparation. LM wrote the manuscript with input from GHFIII and EUP. All authors read, edited, and approved the final manuscript.

\section{Funding}

This work was supported by grants 1R03AR065760 (NIH, NIAMS),

1R21AR068536 (NIH, NIAMS), and MDBR-15-107-DMD (Million Dollar Bike Ride Grant Program) to L.M. L.M. is a member of the Brotman Baty Institute for Precision Medicine (BBI) and this work was funded in part by a Catalytic Collaborations award from the BBI. We would like to thank the University of Washington School of Medicine Medical Student Research Training Program for financial support. Funding bodies played no role in the design of the study, in the collection, analysis, and interpretation of data, or in the writing of the manuscript.

\section{Availability of data and materials}

The datasets used and/or analyzed during the current study are available from the corresponding author on reasonable request.

\section{Ethics approval and consent to participate}

All experiments involving live zebrafish (Danio rerio) were approved by and carried out in compliance with the Institutional Animal Care and Use Committee guidelines of Seattle Children's Research Institute and the University of Maine.

\section{Consent for publication}

Not applicable.

\section{Competing interests}

The authors declare that they have no competing interests.

\section{Author details}

${ }^{1}$ Center for Developmental Biology and Regenerative Medicine, Seattle Children's Research Institute, Seattle, WA, USA. ${ }^{2}$ Medical Student Research Training Program, University of Washington School of Medicine, Seattle, WA, USA. ${ }^{3}$ Molecular Medicine and Mechanisms of Disease Program, Department of Pathology, University of Washington, Seattle, WA, USA. ${ }^{4}$ Department of Pediatrics, University of Washington, Seattle, WA, USA. ${ }^{5}$ Graduate School of Biomedical Science and Engineering, University of Maine, Orono, ME, USA. ${ }^{6}$ School of Biology and Ecology, University of Maine, Orono, ME, USA. 
Received: 16 April 2020 Accepted: 5 October 2020

Published online: 15 October 2020

\section{References}

1. Hoffman EP, Brown RH Jr, Kunkel LM. Dystrophin: the protein product of the Duchenne muscular dystrophy locus. Cell. 1987;51:919-28.

2. Monaco AP, Neve RL, Colletti-Feener C, Bertelson CJ, Kurnit DM, Kunkel LM. Isolation of candidate CDNAs for portions of the Duchenne muscular dystrophy gene. Nature. 1986:323:646-50.

3. Ervasti JM, Ohlendieck K, Kahl SD, Gaver MG, Campbell KP. Deficiency of a glycoprotein component of the dystrophin complex in dystrophic muscle. Nature. 1990;345:315-9.

4. Yoshida M, Ozawa E. Glycoprotein complex anchoring dystrophin to sarcolemma. J Biochem. 1990;108:748-52.

5. Ervasti JM, Campbell KP. A role for the dystrophin-glycoprotein complex as a transmembrane linker between laminin and actin. J Cell Biol. 1993;122:809-23.

6. Allen DG, Whitehead NP, Froehner SC. Absence of dystrophin disrupts skeletal muscle signaling: roles of $\mathrm{Ca} 2+$, reactive oxygen species, and nitric oxide in the development of muscular dystrophy. Physiol Rev. 2016;96:253-305.

7. Spinazzola JM, Kunkel LM. Pharmacological therapeutics targeting the secondary defects and downstream pathology of Duchenne muscular dystrophy. Expert Opin Orphan Drugs. 2016:4:1179-94.

8. Bodensteiner JB, Engel AG. Intracellular calcium accumulation in Duchenne dystrophy and other myopathies: a study of 567,000 muscle fibers in 114 biopsies. Neurology. 1978;28:439-46.

9. Brenman JE, Chao DS, Xia H, Aldape K, Bredt DS. Nitric oxide synthase complexed with dystrophin and absent from skeletal muscle sarcolemma in Duchenne muscular dystrophy. Cell. 1995;82:743-52.

10. Guiraud S, Aartsma-Rus A, Vieira NM, Davies KE, van Ommen GJ, Kunkel LM. The pathogenesis and therapy of muscular dystrophies. Annu Rev Genomics Hum Genet. 2015:16:281-308.

11. Dellorusso C, Crawford RW, Chamberlain JS, Brooks SV. Tibialis anterior muscles in mdx mice are highly susceptible to contraction-induced injury. J Muscle Res Cell Motil. 2001;22:467-75

12. Petrof BJ, Shrager JB, Stedman HH, Kelly AM, Sweeney HL. Dystrophin protects the sarcolemma from stresses developed during muscle contraction. Proc Natl Acad Sci U S A. 1993:90:3710-4.

13. Reeve $J$, McArdle A, Jackson MJ. Age-related changes in muscle calcium content in dystrophin-deficient mdx mice. Muscle Nerve. 1997;20:357-60.

14. Emery AE. Population frequencies of inherited neuromuscular diseases--a world survey. Neuromuscul Disord. 1991;1:19-29.

15. Mah JK, Korngut L, Dykeman J, Day L, Pringsheim T, Jette N. A systematic review and meta-analysis on the epidemiology of Duchenne and Becker muscular dystrophy. Neuromuscul Disord. 2014;24:482-91.

16. Crone M, Mah JK. Current and emerging therapies for Duchenne muscula dystrophy. Curr Treat Options Neurol. 2018;20:31

17. Bushby K, Finkel R, Birnkrant DJ, Case LE, Clemens PR, Cripe L, et al. Diagnosis and management of Duchenne muscular dystrophy, part 1: diagnosis, and pharmacological and psychosocial management. Lancet Neurol. 2010;9:77-93.

18. Kinnett K, Rodger S, Vroom E, Furlong P, Aartsma-Rus A, Bushby K. Imperatives for DUCHENNE MD: a simplified guide to comprehensive care for Duchenne muscular dystrophy. PLoS Curr. 2015;7.

19. Hoffman EP. Pharmacotherapy of Duchenne muscular dystrophy. Handb Exp Pharmacol. 2019 Aug 3. https://doi.org/10.1007/164_2019_256.

20. Chamberlain JR, Chamberlain JS. Progress toward gene therapy for Duchenne muscular dystrophy. Mol Ther. 2017:25:1125-31.

21. Conboy I, Murthy N, Etienne J, Robinson Z. Making gene editing a therapeutic reality [version 1; peer review: 2 approved]. F1000Res. 2018:7:1970.

22. Duan D. Systemic AAV micro-dystrophin gene therapy for Duchenne muscular dystrophyMol Ther. 2018:26:2337-56.

23. Min YL, Bassel-Duby R, Olson EN. CRISPR correction of Duchenne muscular dystrophy. Annu Rev Med. 2019;70:239-55.

24. Guiraud S, Davies KE. Pharmacological advances for treatment in Duchenne muscular dystrophy. Curr Opin Pharmacol. 2017:34:36-48.

25. Consalvi S, Saccone V, Mozzetta C. Histone deacetylase inhibitors: a potential epigenetic treatment for Duchenne muscular dystrophy. Epigenomics. 2014;6:547-60.

26. Bettica P, Petrini S, D'Oria V, D'Amico A, Catteruccia M, Pane M, et al. Histological effects of givinostat in boys with Duchenne muscular dystrophy. Neuromuscul Disord. 2016;26:643-9.
27. Johnson NM, Farr GH 3rd, Maves L. The HDAC inhibitor TSA ameliorates a zebrafish model of Duchenne muscular dystrophy. PLoS Curr. 2013:5: ecurrents.md.8273cf41db10e2d15dd3ab827cb4b027.

28. Minetti GC, Colussi C, Adami R, Serra C, Mozzetta C, Parente V, et al. Functional and morphological recovery of dystrophic muscles in mice treated with deacetylase inhibitors. Nat Med. 2006;12:1147-50.

29. Kouzarides T. Chromatin modifications and their function. Cell. 2007;128:693-705.

30. Xu WS, Parmigiani RB, Marks PA. Histone deacetylase inhibitors: molecular mechanisms of action. Oncogene. 2007;26:5541-52.

31. Chang NC, Sincennes MC, Chevalier FP, Brun CE, Lacaria M, Segalés J, et al. The dystrophin glycoprotein complex regulates the epigenetic activation of muscle stem cell commitment. Cell Stem Cell. 2018;22:755-68.

32. Colussi C, Mozzetta C, Gurtner A, Illi B, Rosati J, Straino S, et al. HDAC2 blockade by nitric oxide and histone deacetylase inhibitors reveals a common target in Duchenne muscular dystrophy treatment. Proc Natl Acad Sci U S A. 2008;105:19183-7.

33. Colussi C, Gurtner A, Rosati J, Illi B, Ragone G, Piaggio G, et al. Nitric oxide deficiency determines global chromatin changes in Duchenne muscular dystrophy. FASEB J. 2009;23:2131-41.

34. Saccone V, Consalvi S, Giordani L, Mozzetta C, Barozzi I, Sandoná M, et al. HDAC-regulated myomiRs control BAF60 variant exchange and direct the functional phenotype of fibro-adipogenic progenitors in dystrophic muscles. Genes Dev. 2014:28:841-57.

35. Colussi C, Banfi C, Brioschi M, Tremoli E, Straino S, Spallotta F, et al. Proteomic profile of differentially expressed plasma proteins from dystrophic mice and following suberoylanilide hydroxamic acid treatment Proteomics Clin Appl. 2010;4:71-83.

36. Consalvi S, Saccone V, Giordani L, Minetti G, Mozzetta C, Puri PL. Histone deacetylase inhibitors in the treatment of muscular dystrophies: epigenetic drugs for genetic diseases. Mol Med. 2011;17:457-65.

37. Consalvi S, Mozzetta C, Bettica P, Germani M, Fiorentini F, Del Bene F, et al. Preclinical studies in the $\mathrm{mdx}$ mouse model of duchenne muscular dystrophy with the histone deacetylase inhibitor givinostat. Mol Med. 2013:19:79-87.

38. Bajanca F, Vandel L. Epigenetic regulators modulate muscle damage in Duchenne muscular dystrophy model. PLoS Curr. 2017 Dec;21:9.

39. Bassett DI, Bryson-Richardson RJ, Daggett DF, Gautier P, Keenan DG, Currie PD. Dystrophin is required for the formation of stable muscle attachments in the zebrafish embryo. Development. 2003;130:5851-60.

40. Bassett D, Currie PD. Identification of a zebrafish model of muscular dystrophy. Clin Exp Pharmacol Physiol. 2004;31:537-40.

41. Berger J, Berger S, Hall TE, Lieschke GJ, Currie PD. Dystrophin-deficient zebrafish feature aspects of the Duchenne muscular dystrophy pathology. Neuromuscul Disord. 2010;20:826-32.

42. Maves L. Recent advances using zebrafish animal models for muscle disease drug discovery. Expert Opin Drug Discov. 2014;14:1-13.

43. Widrick JJ, Kawahara G, Alexander MS, Beggs AH, Kunkel LM. Discovery of novel therapeutics for muscular dystrophies using zebrafish phenotypic screens. J Neuromuscul Dis. 2019:6:271-87.

44. Granato M, van Eeden FJ, Schach U, Trowe T, Brand M, Furutani-Seiki M, et al. Genes controlling and mediating locomotion behavior of the zebrafish embryo and larva. Development. 1996;123:399-413.

45. Kawahara G, Karpf JA, Myers JA, Alexander MS, Guyon JR, Kunkel LM. Drug screening in a zebrafish model of Duchenne muscular dystrophy. Proc Natl Acad Sci U S A. 2011:108:5331-6.

46. Sztal TE, Ruparelia AA, Williams C, Bryson-Richardson RJ. Using touch-evoked response and locomotion assays to assess muscle performance and function in zebrafish. J Vis Exp. 2016;54431.

47. Berger J, Sztal T, Currie PD. Quantification of birefringence readily measures the level of muscle damage in zebrafish. Biochem Biophys Res Commun. 2012:423:785-8.

48. Kawahara G, Gasperini MJ, Myers JA, Widrick JJ, Eran A, Serafini PR, et al. Dystrophic muscle improvement in zebrafish via increased heme oxygenase signaling. Hum Mol Genet. 2014;23:1869-78.

49. Waugh TA, Horstick E, Hur J, Jackson SW, Davidson AE, Li X, et al. Fluoxetine prevents dystrophic changes in a zebrafish model of Duchenne muscular dystrophy. Hum Mol Genet. 2014;23:4651-62.

50. Hightower RM, Reid AL, Gibbs DE, Wang Y, Widrick JJ, Kunkel LM, et al. The SINE compound KPT-350 blocks dystrophic pathologies in DMD zebrafish and mice. Mol Ther 2020:28:189-201. 
51. Li M, Andersson-Lendahl M, Sejersen T, Arner A. Muscle dysfunction and structural defects of dystrophin-null sapje mutant zebrafish larvae are rescued by ataluren treatment. FASEB J. 2014;28:1593-9.

52. Winder SJ, Lipscomb L, Angela Parkin C, Juusola M. The proteasomal inhibitor MG132 prevents muscular dystrophy in zebrafish. PLoS Curr. 2011; 3:RRN1286.

53. Westerfield M. The zebrafish book, 5th edition; a guide for the laboratory use of zebrafish (Danio rerio); 2007.

54. Berger J, Berger S, Jacoby AS, Wilton SD, Currie PD. Evaluation of exonskipping strategies for Duchenne muscular dystrophy utilizing dystrophindeficient zebrafish. J Cell Mol Med. 2011;15:2643-51.

55. Smith LL, Beggs AH, Gupta VA. Analysis of skeletal muscle defects in larval zebrafish by birefringence and touch-evoke escape response assays. J Vis Exp. 2013;50925

56. Goody MF, Kelly MW, Reynolds CJ, Khalil A, Crawford BD, Henry CA. NAD+ biosynthesis ameliorates a zebrafish model of muscular dystrophy. PLoS Biol. 2012;10:e1001409.

57. Guyon JR, Goswami J, Jun SJ, Thorne M, Howell M, Pusack T, et al. Genetic isolation and characterization of a splicing mutant of zebrafish dystrophin Hum Mol Genet. 2009;18:202-11.

58. Lipscomb L, Piggott RW, Emmerson T, Winder SJ. Dasatinib as a treatment for Duchenne muscular dystrophy. Hum Mol Genet. 2016;25:266-74.

59. Huber K, Doyon G, Plaks J, Fyne E, Mellors JW, Sluis-Cremer N. Inhibitors of histone deacetylases: correlation between isoform specificity and reactivation of HIV type 1 (HIV-1) from latently infected cells. J. Biol. Chem. 2011;286:22211-8

60. Kim YB, Lee KH, Sugita K, Yoshida M, Horinouchi S. Oxamflatin is a novel antitumor compound that inhibits mammalian histone deacetylase. Oncogene. 1999;18:2461-70.

61. Lara E, Mai A, Calvanese V, Altucci L, Lopez-Nieva P, Martinez-Chantar ML, et al. Salermide, a sirtuin inhibitor with a strong cancer-specific proapoptotic effect. Oncogene. 2009;28:781-91.

62. Vaquero A, Scher $M$, Lee D, Erdjument-Bromage $H$, Tempst $P$, Reinberg D. Human SirT1 interacts with histone $\mathrm{H} 1$ and promotes formation of facultative heterochromatin. Mol Cell. 2004;16:93-105.

63. Vaquero A, Scher MB, Lee DH, Sutton A, Cheng HL, Alt FW, et al. SirT2 is a histone deacetylase with preference for histone H4 Lys 16 during mitosis. Genes Dev. 2006:20:1256-61.

64. Sonoda H, Nishida K, Yoshioka T, Ohtani M, Sugita K. Oxamflatin: a novel compound which reverses malignant phenotype to normal one via induction of JunD. Oncogene. 1996;13:143-9.

65. Wang YL, Liui HL, Fu RG, Wang ZW, Ren HT, Dai ZJ, et al. HDAC inhibitor oxamflatin induces morphological changes and has strong cytostatic effects in ovarian cancer cell lines. Curr Mol Med. 2016;16:232-42.

66. Liu G, Su L, Hao X, Zhong N, Zhong D, Singhal S, et al. Salermide upregulates death receptor 5 expression through the ATF4-ATF3-CHOP axis and leads to apoptosis in human cancer cells. J Cell Mol Med. 2012;16: $1618-28$.

67. Rotili D, Tarantino D, Nebbioso A, Paolini C, Huidobro C, Lara E, et al. Discovery of salermide-related sirtuin inhibitors: binding mode studies and antiproliferative effects in cancer cells including cancer stem cells. J Med Chem. 2012;55:10937-47

68. Bosch-Presegué L, Vaquero A. Sirtuin-dependent epigenetic regulation in the maintenance of genome integrity. FEBS J. 2015;282:1745-67.

69. Chalkiadaki A, Igarashi M, Nasamu AS, Knezevic J, Guarente L. Musclespecific SIRT1 gain-of-function increases slow-twitch fibers and ameliorates pathophysiology in a mouse model of Duchenne muscular dystrophy. PLoS Genet. 2014;10:e1004490.

70. Pasco MY, Rotili D, Altucci L, Farina F, Rouleau GA, Mai A, et al. Characterization of sirtuin inhibitors in nematodes expressing a muscular dystrophy protein reveals muscle cell and behavioral protection by specific sirtinol analogues. J Med Chem. 2010;53:1407-11.

71. Keskin-Aktan A, Akbulut KG, Yazici-Mutlu Ç, Sonugur G, Ocal M, Akbulut H. The effects of melatonin and curcumin on the expression of SIRT2, BCl-2 and Bax in the hippocampus of adult rats. Brain Res Bull. 2018;137:306-10.

72. Shi $Y, X u X$, Zhang Q, Fu G, Mo Z, Wang GS, et al. tRNA synthetase counteracts c-Myc to develop functional vasculature. Elife. 2014;3:e02349.

73. Spinazzola JM, Lambert MR, Gibbs DE, Conner JR, Krikorian GL, Pareek P, et al. Effect of serotonin modulation on dystrophin-deficient zebrafish. Biol Open. 2020:9:bio053363.
74. Choi IY, Lim H, Estrellas K, Mula J, Cohen TV, Zhang Y, et al. Concordant but varied phenotypes among Duchenne muscular dystrophy patient-specific myoblasts derived using a human iPSC-based model. Cell Rep. 2016;15: 2301-12.

75. McGreevy JW, Hakim CH, Mclntosh MA, Duan D. Animal models of Duchenne muscular dystrophy: from basic mechanisms to gene therapy. Dis Model Mech. 2015;8:195-213.

76. Wells DJ. Tracking progress: an update on animal models for Duchenne muscular dystrophy. Dis Model Mech. 2018;11:dmm035774.

77. Consalvi S, Sandoná M, Saccone V. Epigenetic reprogramming of muscle progenitors: inspiration for clinical therapies. Stem Cells Int. 2016;2016: 6093601.

78. Campbell AE, Oliva J, Yates MP, Zhong JW, Shadle SC, Snider L, et al. BET bromodomain inhibitors and agonists of the beta- 2 adrenergic receptor identified in screens for compounds that inhibit DUX4 expression in FSHD muscle cells. Skelet Muscle. 2017;7:16.

79. Gordon BS, Delgado Díaz DC, Kostek MC. Resveratrol decreases inflammation and increases utrophin gene expression in the mdx mouse model of Duchenne muscular dystrophy. Clin Nutr. 2013;32:104-11.

80. Riessland M, Ackermann B, Förster A, Jakubik M, Hauke J, Garbes L, et al. SAHA ameliorates the SMA phenotype in two mouse models for spinal muscular atrophy. Hum Mol Genet. 2010;19:1492-506.

81. Abdelfatah E, Kerner Z, Nanda N, Ahuja N. Epigenetic therapy in gastrointestinal cancer: the right combination. Therap Adv Gastroenterol. 2016;9:560-79.

82. Ahuja N, Sharma AR, Baylin SB. Epigenetic therapeutics: a new weapon in the war against cancer. Annu Rev Med. 2016;67:73-89.

83. Bai Y, Li W, Wang T, Ahmad D, Cui G. Research advances in the use of histone deacetylase inhibitors for epigenetic targeting of cancer. Curr Top Med Chem. 2019. https://doi.org/10.2174/1568026619666190125145110.

84. Alexanian M, Padmanabhan A, McKinsey TA, Haldar SM. Epigenetic therapies in heart failure. J Mol Cell Cardiol. 2019;130:197-204.

85. Mazur PK, Herner A, Mello SS, Wirth M, Hausmann S, Sánchez-Rivera FJ, et al. Combined inhibition of BET family proteins and histone deacetylases as a potential epigenetics-based therapy for pancreatic ductal adenocarcinoma. Nat Med. 2015;21:1163-71

\section{Publisher's Note}

Springer Nature remains neutral with regard to jurisdictional claims in published maps and institutional affiliations.
Ready to submit your research? Choose BMC and benefit from:
- fast, convenient online submission
- thorough peer review by experienced researchers in your field
- rapid publication on acceptance
- support for research data, including large and complex data types
- gold Open Access which fosters wider collaboration and increased citations
- maximum visibility for your research: over $100 \mathrm{M}$ website views per year
At $\mathrm{BMC}$, research is always in progress. 